Martín-Seijo, M, y Vázquez Collazo, S. (2020): “Ad comburendum. El uso de la leña en el Castillo de Pambre (Palas de Rei, Lugo) durante los siglos XV-XVII", Spal 29.1: 321-341.

DOI: http://dx.doi.org/10.12795/spal.2020.i29.12

\title{
AD COMBURENDUM. EL USO DE LA LEÑA EN EL CASTILLO DE PAMBRE (PALAS DE REI, LUGO) DURANTE LOS SIGLOS XV-XVII
}

\section{AD COMBURENDUM. FIREWOOD USE IN THE PAMBRE CASTLE (PALAS DE REI, LUGO) DURING THE 15TH-17TH CENTURIES AD}

\author{
MARÍA MARTÍN-SEIJO \\ Responsable de la correspondencia \\ Grupo de Estudos para a Prehistoria do NO Ibérico-Arqueoloxía, Antigüidade e Territorio. GEPN-AAT (GI-1534). \\ Departamento de Historia. Universidade de Santiago de Compostela. Praza da Universidade 1. 15782. Santiago de Compostela, Spain \\ Correo-e: maria.martin.seijo@usc.es. (D) https://orcid.org/0000-0003-2924-7763 \\ Researcher ID: <https://publons.com/researcher/M-2214-2013> \\ SANTIAGO VÁZQUEZ COLLAZO \\ TOMOS Conservación-Restauración S.L. Rúa San Agustín, 28 - 4․ 15001 - A Coruña \\ Correo-e: tomos@tomos.es. (D) https://orcid.org/0000-0002-3941-0731
}

Resumen: Se presentan los resultados del análisis antracológico, así como los datos dendrológicos y tafonómicos de varias muestras de carbón. Estas fueron recuperadas durante la intervención arqueológica desarrollada en el Castillo de Pambre (Palas de Rei, Lugo) en el año 2014, en relación con las tareas de rehabilitación del conjunto de edificaciones del castillo. Los datos obtenidos nos permiten definir el uso como combustible de los recursos leñosos que realizaron los ocupantes del castillo durante los siglos XV-XVII. Se documenta un predominio de la madera de roble (Quercus sp. caducifolio), seguida del avellano (Corylus avellana) junto con una gran diversidad de taxones, especialmente de árboles frutales, mientras que los taxones de matorral son poco significativos en el conjunto. Esto a pesar de coincidir con un período frío, la Pequeña Edad del Hielo, y de la importante deforestación registrada tanto en los datos paleoambientales como en la documentación.

Palabras clave: Antracología, madera, frutales, Pequeña Edad del Hielo, Edad Moderna, fortificaciones.

\begin{abstract}
This paper presents the results of charcoal analysis as well as dendrological and taphonomical attributes of charcoal samples. These were recovered during the archaeological survey in the Pambre Castle (Palas de Rei, Lugo) during 2014 and in relation to the rehabilitation activities in the group of buildings of the castle. The data obtained let us to define the use as firewood of wood resources performed by the inhabitants of the castle between $15^{\text {th }}$ to $17^{\text {th }}$ centuries AD. A predominance of oak (deciduous Quercus), followed by hazel wood (Corylus avellana) has been attested, besides a great taxa diversity, predominantly fruit trees whilst scrubland taxa are not significant in the assemblage. This even though it coincided with a cold period, the Little Ice Age, and the major deforestation registered in the paleoenvironmental proxies as well as in the written sources.
\end{abstract}

Keywords: Charcoal analysis, wood, fruit trees, Little Ice Age, Post-medieval, fortifications. 


\section{INTRODUCCIÓN}

Durante la Edad Moderna al igual que en la Edad Media (Le Goff 2002: 178, Pastoreau 2006, Fossier 2017), la madera continuó siendo la materia prima por excelencia. A pesar de que las plantas y las materias primas derivadas de estas cumplían múltiples finalidades como la obtención de alimento y forraje, combustible, y eran utilizadas en la construcción de estructuras y edificaciones, así como en la manufactura de todo tipo de artefactos incluyendo aquellos relacionados con el uso personal, herramientas, armas y medios de transporte, la aplicación de la antracología en este tipo de contextos de cronología reciente es muy puntual. Un hecho que es especialmente notorio en el análisis de muestras procedentes de contextos datados en el período de transición entre la Edad Media y la Edad Moderna. Este vacío en la investigación no solo afecta al noroeste peninsular (Martín-Seijo et al. 2010), sino que también es observable en el ámbito ibérico (Euba 2008, 2009, 2010, Cubero 2011, Ntinou et al. 2013, Mira et al. 2017) e incluso en el europeo (Zutter 2000, Leonardi 2002, Scipioni 2002, Ludemann et al. 2004, Burri 2010, Huntley 2010, Py y Durand 2010, Euba et al. 2011, Warren et al. 2012, Deforce et al. 2013, Knapp et al. 2015, Hazel et al. 2017, Burri 2018).

Los datos antracológicos de estas cronologías tardías proceden, de forma predominante, de estructuras relacionadas con la producción de carbón vegetal. Hasta el momento los únicos datos antracológicos para estas cronologías en el noroeste peninsular serían los del asentamiento rural de A Mourela (As Pontes, A Coruña). En este yacimiento se documentaron varios momentos de ocupación, y entre ellos uno datado entre los siglos XIII-XVII d.n.e.- (Martín-Seijo et al. 2009, 2010). Otros datos sobre los usos de la madera durante este período han sido obtenidos a partir del análisis xilológico de los objetos manufacturados preservados por anegamiento en el puerto de Pontevedra (Martín-Seijo et al. 2018). A nivel peninsular y en relación con el estudio de la madera, para este período también se han realizado análisis dendocronológicos, de dendroproveniencia y químicos en relación con pecios sumergidos (Traoré et al. 2017, Traoré 2018) o con maderas utilizadas en la construcción de edificios religiosos (Domínguez-Delmás et al. 2018).

A pesar de la escasez de estudios de carbones y maderas en contextos de esta cronología, los datos que pueden proporcionar este tipo de análisis arqueobotánicos pueden llegar a ser muy relevantes. La antracología permite completar y contrastar la información disponible en otros archivos medioambientales y proporciona datos relacionados con la vida cotidiana que son difíciles de rastrear a partir del estudio de las fuentes escritas. Este es el caso de la gestión de los recursos leñosos para la obtención de combustibles, y del aprovechamiento del bosque. Los estudios de la documentación escrita tanto de época medieval como de época moderna señalan una sobrerrepresentación en las fuentes documentales de aquellos árboles que eran objeto de un aprovechamiento directo, mientras que otros árboles y arbustos son invisibles en las fuentes, por no haber sido protegidos por la legislación o por no haber sido utilizados para pagar rentas (Carlé 1976, López-Sabatel 2009, Devia 2015). El estudio de muestras arqueobotánicas nos remite directamente a la importancia que el bosque tenía para estas comunidades y los esfuerzos destinados a proteger los árboles y las formaciones forestales (Carlé 1976), en un momento de fuerte deforestación (Guitián Rivera 2001), relacionado con la apertura de campos de cultivo pero también con la obtención de combustible destinado al abastecimiento de madera, leña y carbón de las ciudades (Armas Castro 1992, Franco-Rubio 2009, Estrella y Asla 2010), así como más tarde a la producción de hierro (González-Pérez 1994) y al aprovisionamiento de madera para la industria naval (Gomes y Gomes 2016). Por otra parte, el estudio de muestras arqueobotánicas de estas cronologías también es relevante porque coincide con un período de inestabilidad climática (1300-1850) conocido como la Pequeña Edad del Hielo (Fagan 2008) y que es considerado uno de los períodos más fríos del Holoceno reciente (Oliva et al. 2018).

El primer objetivo de este trabajo ha sido intentar aproximarnos a la cotidianeidad de las comunidades pasadas a partir de uno de los residuos más habituales generados por las actividades cotidianas: los carbones. Los carbones nos permiten aproximarnos a la vida diaria en el castillo, fundamentalmente y por los contextos en los que han sido recuperadas las muestras, al tipo de leña utilizada para el procesado de alimentos o para la iluminación y la calefacción de las estancias. La caracterización de la leña consumida incluirá no solo la identificación taxonómica sino también el registro de aspectos dendrológicos y tafonómicos que permitan completar datos como el tipo de leña, la existencia o no de almacenaje, etc. La leña nos permite conectar también esta actividad desarrollada intramuros con el exterior, con el bosque, la orla boscosa u otras formaciones vegetales en las que se producía el aprovisionamiento de dichos combustibles. Incluso y por la existencia de fuentes históricas para este período, podríamos 
plantearnos identificar quiénes fueron los encargados del aprovisionamiento de la leña y cómo era el acceso a este tipo de recursos leñosos. El segundo objetivo será obtener información paleoambiental a partir de los recursos leñosos explotados por estas comunidades, lo que nos permitirá completar los datos antracológicos y polínicos disponibles hasta el momento. Esta aproximación es cualitativa y su interpretación paleoambiental se encuentra limitada por las propias características del conjunto arqueobotánico; no obstante, sí se pueden identificar las especies arbóreas y arbustivas existentes en el entorno del castillo y utilizadas como leña.

\section{CONTEXTO ARQUEOLÓGICO, HISTÓRICO Y AMBIENTAL}

El Castillo de Pambre se encuentra localizado en el suroeste de la provincia de Lugo, Concello de Palas de Rei, parroquia de San Pedro de Pambre (fig. 1). La Fortaleza de Pambre es uno de los mejores ejemplos de la arquitectura militar de la Edad Media en Galicia (fig. 2). Su construcción se remonta a los años finales del siglo XIV o inicios del siglo XV y está atribuida a Gonzalo Ozores de Ulloa (Pardo de Guevara et al. 2011-2012, Rouco 2017). Lope Sánchez de Ulloa construyó el primer recinto y lo enriqueció, y su nieto Sancho Sánchez de Ulloa realizó el segundo y el reacondicionamiento palaciego en el interior del primero (Pardo de Guevara et al. 2011-2012, Rouco 2017). Estas dos fases constructivas son visibles en la propia estructura de la fortificación, con una torre del homenaje y dos recintos que la rodean, aunque no parecen observarse grandes diferencias arquitectónicas y probablemente la diferencia cronológica entre ambas fue escasa (Pardo de Guevara et al. 2011-2012). En el interior de uno de los recintos queda englobada una capilla románica datada en el siglo XI y posteriormente remodelada. Cuando la fortaleza se destina a usos agrícolas, hacia el siglo XVI, se construyen toda una serie de edificaciones con diferentes técnicas constructivas y materiales (Rouco 2017). El conjunto palaciego funciona como residencia habitual de Inés de Castro y de su hijo Sancho de Ulloa durante el s. XV, pasando a ser la residencia habitual de los Ulloa (Rouco 2007). Esta función decae tras la muerte de Sancho en 1505, momento a partir del que el castillo servirá como núcleo de una extensa explotación económica (Rouco 2017).

Este recinto fortificado ocupa un pequeño promontorio rocoso situado a $430 \mathrm{~m}$ s.n.m., a la orilla del río Pambre, que le sirve de defensa natural rodeándolo en sus caras norte y oeste. Actualmente este yacimiento se encuentra rodeado de áreas de pasto, formaciones de matorral en las zonas más elevadas situadas al norte y bosques de ribera a lo largo del río. En el entorno inmediato se han identificado varios ejemplares de roble (Quercus robur, Q. petraea) y castaño (Castanea sativa). En zonas próximas se localizan plantaciones forestales de pinos y eucaliptos. El Castillo de Pambre se encuentra en el piso colino de la región eurosiberiana, donde actualmente las formaciones arboladas más características serían los bosques mixtos planocaducifolios, con el roble (Quercus robur, $Q$. petraea, $Q$. pyrenaica) como especie predominante, acompañado de abedules (Betula alba, B. pendula), fresnos (Fraxinus excelsior), álamos (Populus tremula) o arces (Acer pseudoplatanus, A. platanoides) entre otros, y también pueden estar presentes plantas perennes como el acebo (Ilex aquifolium), el alcornoque (Quercus suber), la encina (Quercus ilex, Quercus rotundifolia) o el laurel (Laurus nobilis) (Costa et al. 2001, Amigo et al. 2017). La principal característica de estas formaciones es la gran riqueza de especies arbóreas y arbustivas acompañantes: Castanea sativa, Frangula alnus, Prunus avium, P. padus, Crataegus monogyna, Corylus avellana, Juniperus commnunis, etc. (Costa et al. 2001). Los matorrales de sustitución de estas formaciones están formados fundamentalmente por especies de la familia de las Ericaceae y de las Fabaceae (Ulex sp., Genista sp.) (Amigo et al. 2017). Las formaciones riparias de la región eurosiberiana están formadas por alisos (Alnus glutinosa), álamos temblones (Populus tremula), fresnos (Fraxinus excelsior), laureles (Laurus nobilis), abedules (Betula pendula) o diversos tipos de sauces (Salix spp.) (Costa et al. 2001).

Gran parte de la ocupación del castillo coincide con la Pequeña Edad del Hielo (1300-1850), un período frío en el que se han distinguido diferentes fases y cuyas evidencias han sido documentadas tanto en el registro paleoambiental del noroeste (Martínez-Cortizas et al. 1999, Desprat et al. 2003) como en las fuentes históricas (Fernández-Cortizo 2016). Para el período que nos interesa en relación con la ocupación del Castillo de Pambre, entre el 1300 y el 1480 se documenta un enfriamiento creciente con oscilaciones climáticas moderadas, entre 1480 y 1570 se dan unas condiciones más templadas, seguidas posteriormente de un enfriamiento gradual entre 1570 y 1620 , llegando al período más frío conocido como el Mínimo de Maunder entre los años 1620 y 1715 , con temperaturas aproximadamente $2^{\circ} \mathrm{C}$ por debajo de las actuales 


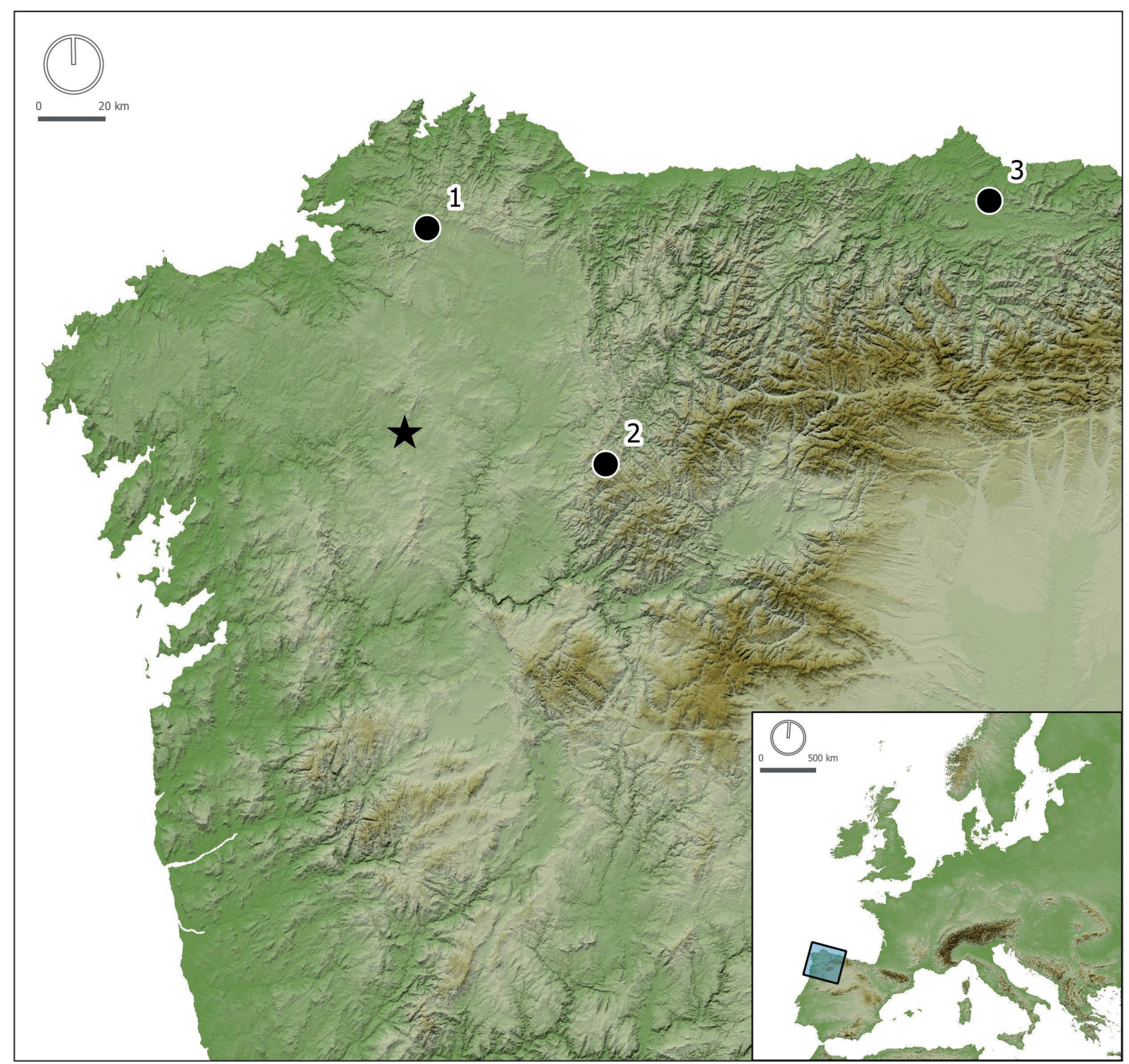

Figura 1. Localización del Castillo de Pambre (Palas de Rei, Lugo) en el noroeste peninsular (estrella), y otros yacimientos citados en el texto: 1) A Mourela, 2) Cova Eirós y 3) Peñaferruz (mapa elaborado por Emilio Abad Vidal).

(Oliva et al. 2018). A partir de los análisis dendroclimáticos, los períodos que concentran una mayor cantidad de oscilaciones se producirían en los intervalos comprendidos entre el 1400 y c 1600 y las temperaturas alcanzarían sus valores más bajos en el 1500 (Manrique y Fernández Cancio 2000). En el noroeste de Iberia los registros documentados en las turberas y en los depósitos marinos señalan el año 1700 como el momento en el que se alcanzan los episodios más fríos de la Pequeña Edad del Hielo (Martínez-Cortizas et al. 1999, Desprat et al. 2003).

\section{MATERIAL Y MÉTODOS}

Han sido analizados 806 fragmentos de carbón recuperados de tres muestras de sedimento, que fueron recogidas, cribadas y triadas para la realización de análisis zooarqueológicos en la Universidad de León. El volumen inicial de las muestras recogidas en campo fue de entre 7,8 y 11,31 litros, de los que se cribó aproximadamente la mitad, recuperándose carbón en todas ellas (tab. 1).

Todas las muestras fueron recogidas en un perfil del área de excavación situada frente a la puerta de la torre 


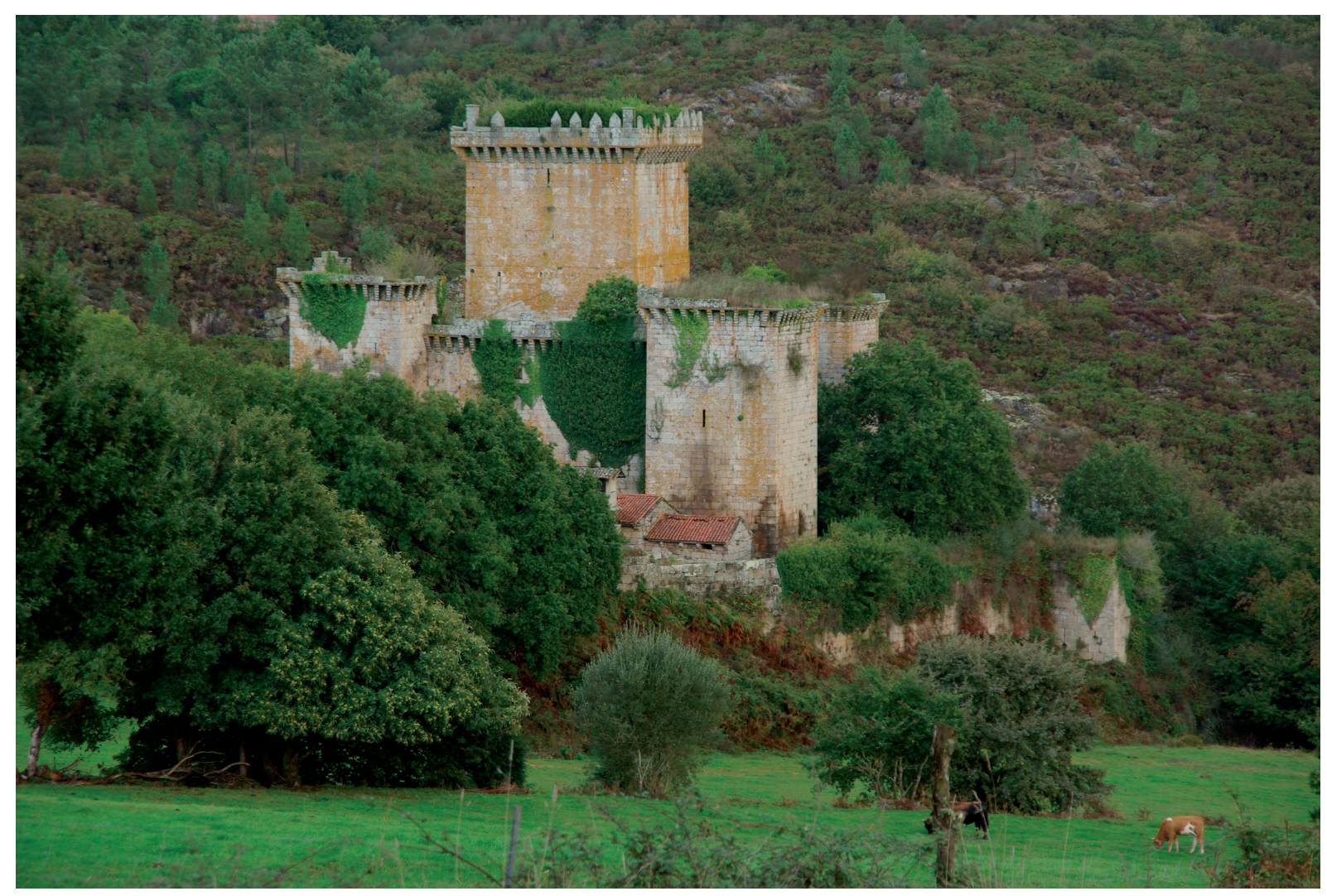

Figura 2. Vista del Castillo de Pambre desde el sur (fotografía: Santiago Vázquez Collazo).

Tabla 1. Resumen de los datos de las tres muestras analizadas.

\begin{tabular}{|c|c|c|c|}
\hline Código & Volumen inicial (1) & Volumen cribado (l) & Peso carbón (gr) \\
\hline M-0 & 7,90 & 3,05 & 91,80 \\
\hline M-1 & 7,80 & 3,06 & 34,64 \\
\hline M-2 & 11,31 & 4,76 & 22,60 \\
\hline
\end{tabular}

Las muestras están depositadas en el Laboratorio de Arqueobotánica del GEPN-AAT en la Universidade de Santiago de Compostela.

de homenaje del castillo de Pambre (fig. 3). La torre estaba situada en la zona central del recinto y su puerta, situada en la fachada norte, era elevada y se localizaba en la primera planta (Rouco 2017). Los carbones estaban relacionados con un depósito interpretado como basurero y están datados a partir de los materiales asociados, fundamentalmente cerámica y monedas, entre los siglos XV-XVI, pudiendo llegar incluso a inicios del siglo XVII.

Durante el estudio microscópico de las muestras de carbón, cada uno de los fragmentos fue identificado taxonómicamente siguiendo el procedimiento habitual (Cartwright 2015). Las muestras fueron observadas en un microscopio Olympus CX-40 con objetivos de $2 \mathrm{x}, 4 \mathrm{x}, 10 \mathrm{x}, 20 \mathrm{x}$ y $40 \mathrm{x}$ aumentos y sus características anatómicas, comparadas con bibliografía especializada (Schweingruber 1990, Gale y Cutler 2000, Hather 2000). Las fotografías fueron realizadas con una lupa binocular Olympus SZX7.

En paralelo al análisis antracológico se registraron también atributos dendrológicos y tafonómicos, con el objetivo de poder complementar los datos obtenidos de la identificación taxonómica. Se ha documentado la curvatura del anillo de crecimiento, una característica cualitativa que nos proporciona un valor relativo sobre el calibre de la madera consumida y la disposición 


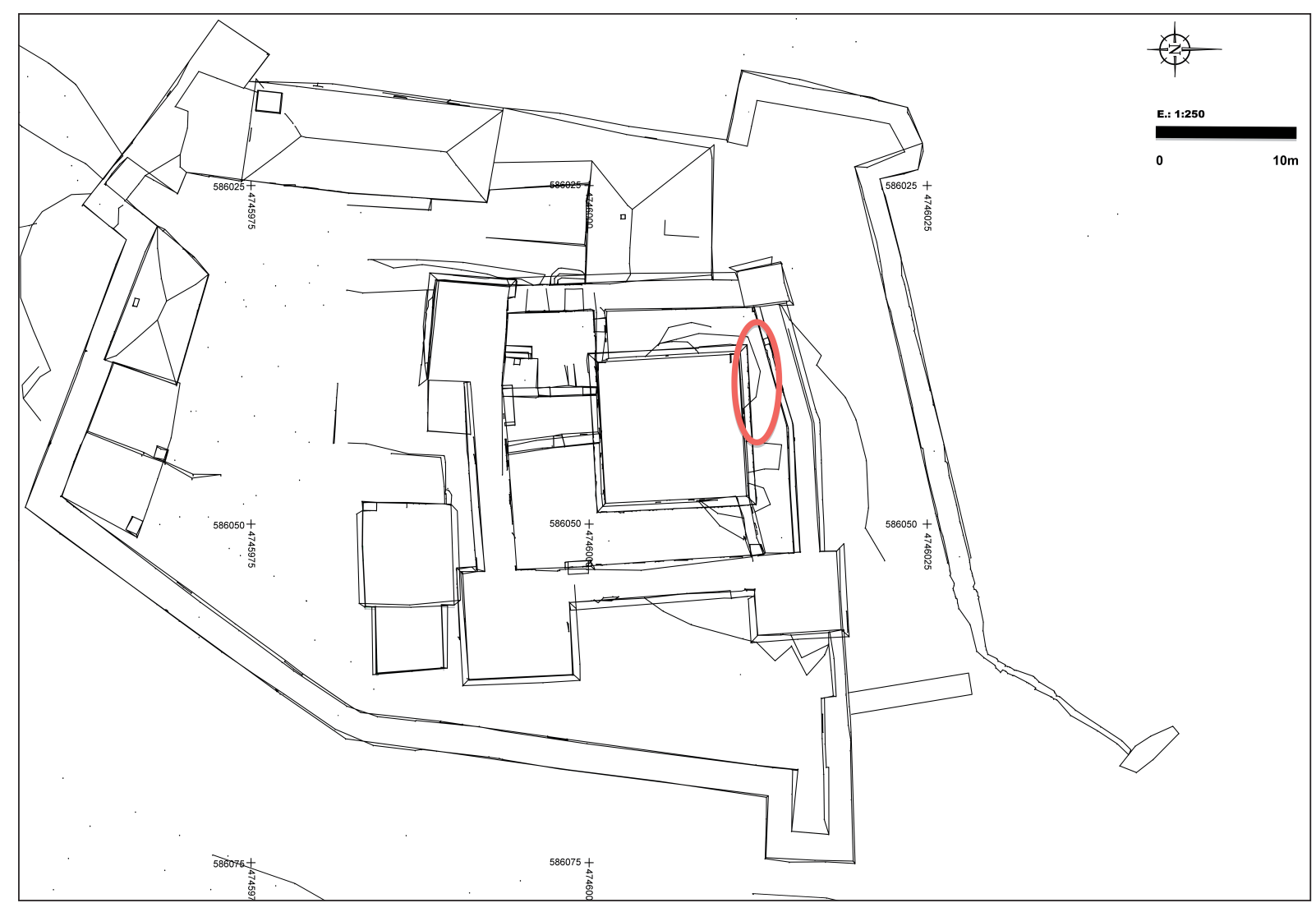

Figura 3. Plano del castillo de Pambre y en rojo, procedencia de las muestras analizadas.

del fragmento en el tronco. La curvatura del anillo indica si la madera procede de la parte central o exterior del tronco, si se trata de un tronco de calibre medio o grande, o de una pequeña rama (Carrión 2006, Marguerie y Hunot 2007) (fig. 4).

Se han registrado también alteraciones anatómicas relacionadas con las condiciones de vida de la planta, como la existencia de variaciones del ritmo normal de crecimiento. Esta es identificada por la supresión del crecimiento del leño final en los anillos anuales, conocida como narrow rings, y puede estar relacionada con la existencia de prácticas de poda, o con períodos desfavorables para el crecimiento de la planta (Schweingruber 2007, Schweingruber y Börner 2018). El ataque de microorganismos a plantas leñosas desencadena mecanismos de defensa destinados a detener su avance en el interior del árbol, en un proceso conocido como CODIT- Compartmentalization of Decay in Trees (fig. 5c) (Shigo y Marx 1977, Shigo 1979, Schweingruber y Börner 2018). Se puede documentar también la existencia de tejido cicatricial en el leño, una reacción a la existencia de algún tipo de herida en el cambium, cuyo origen puede ser muy diverso y difícil de establecer debido a la fragmentación de carbones estudiados habitualmente en las muestras antracológicas (Schweingruber 2007, Schweingruber y Börner 2018). Finalmente, se han registrado también otras evidencias relacionadas con la degradación biológica de la madera (Hickin 1963), como la presencia de hifas de hongos o galerías de insectos xilófagos (Carrión 2006, Marguerie y Hunot 2007, Moskal-del-Hoyo et al. 2010, Fohrer et al. 2017, Toriti 2018).

Se registraron también alteraciones relacionadas con el proceso de combustión, como la presencia de grietas y la vitrificación. Las grietas (radiales, tangenciales o arbitrarias) son habituales en las maderas y carbones arqueológicos (fig. 5a); su presencia está muy condicionada por la propia estructura anatómica de cada especie: afecta principalmente a aquellas maderas que presentan una importante densidad de radios o a aquellas que presentan radios multiseriados, y se localizan normalmente en las zonas más alejadas del duramen (Théry-Parisot y Henry 2012). La vitrificación es un tipo de alteración que hace que los carbones 


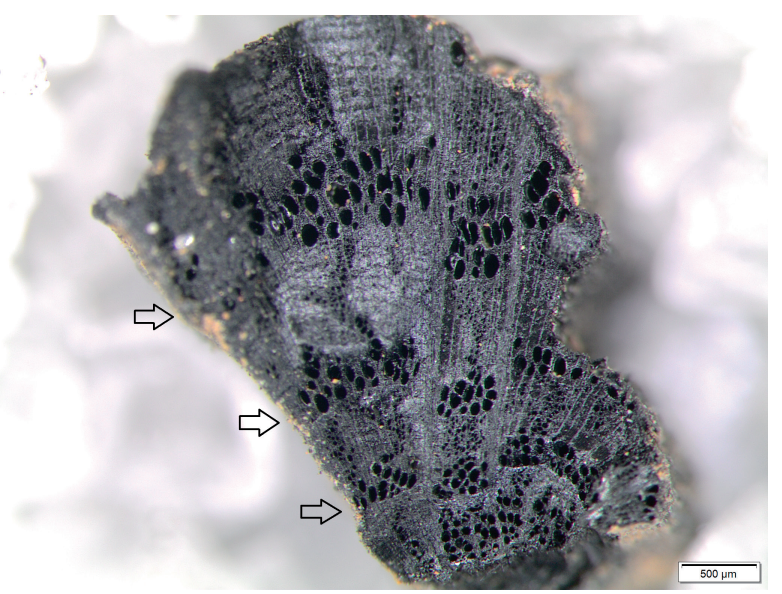

a

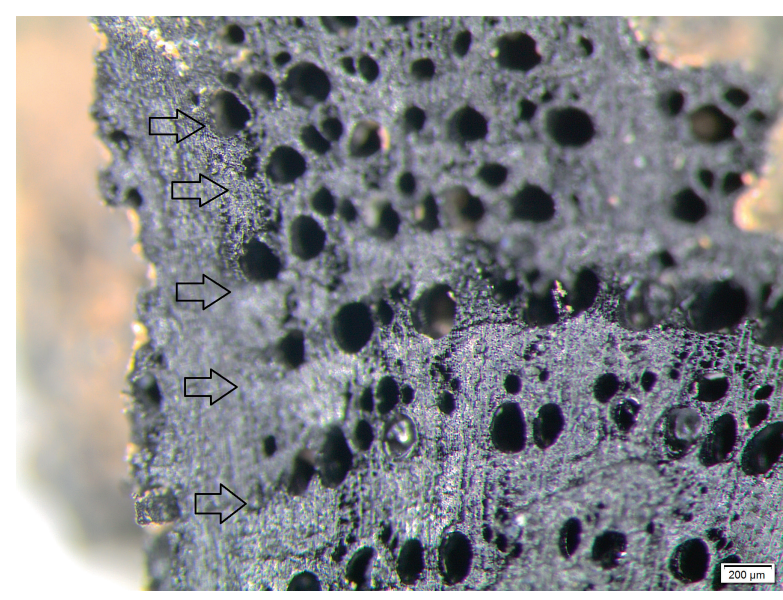

b

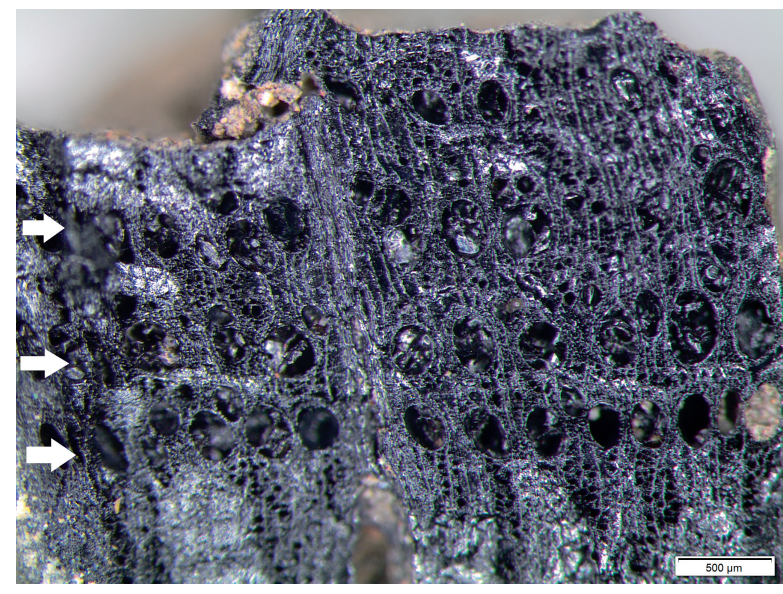

c

Figura 4. Curvatura del anillo de crecimiento en tres fragmentos de Quercus sp. caducifolio de Pambre: a) curvatura fuerte, b) moderada y c) débil.

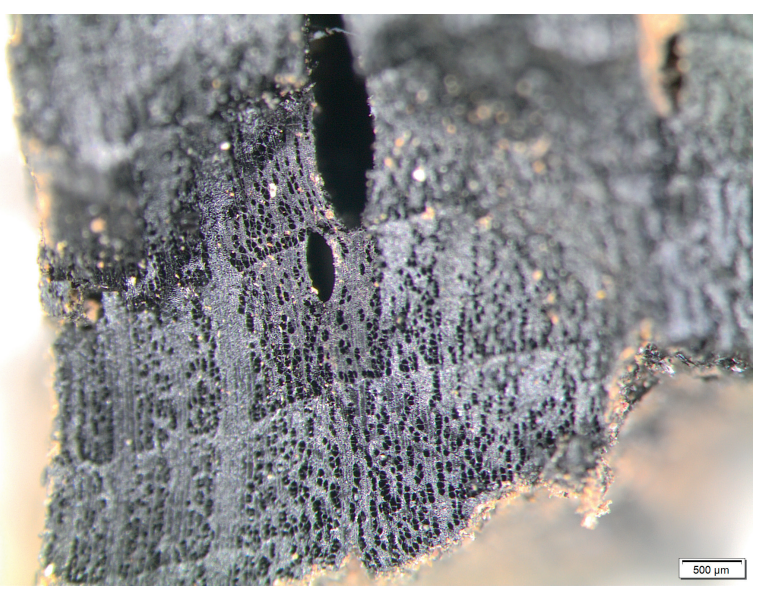

a

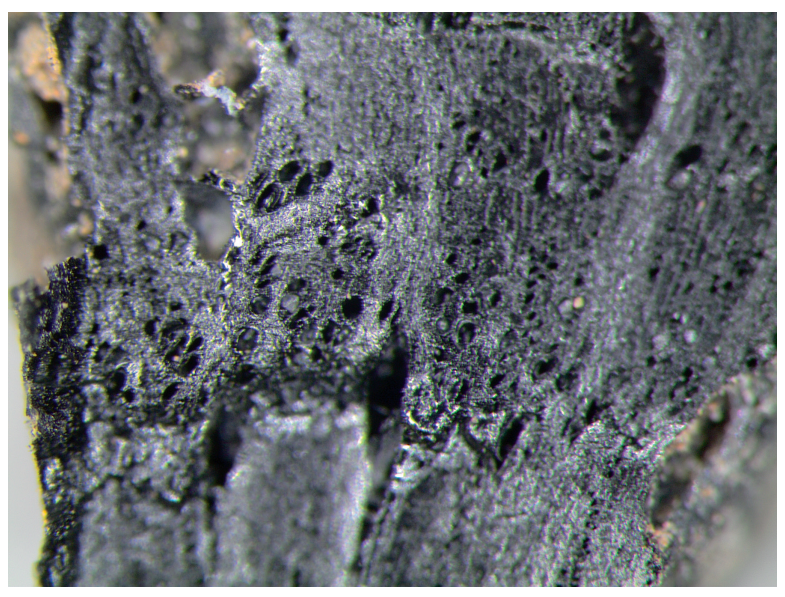

b

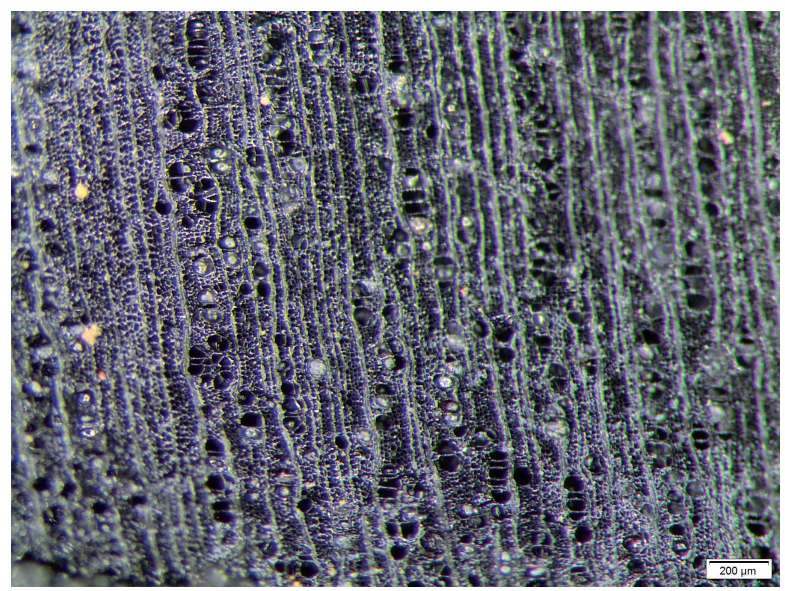

c

Figura 5. Alteraciones relacionadas con el proceso de combustión: a) grietas radiales en Corylus avellana, b) grietas radiales y vitrificación de los tejidos en Quercus sp. Caducifolio. Alteraciones relacionadas con la vida de la planta: c) presencia de compartimentalización en Corylus avellana. 
Tabla 2. Resumen de los resultados antracológicos obtenidos en tres muestras recuperadas en el castillo de Pambre.

\begin{tabular}{|c|c|c|c|c|c|}
\hline Formación & Taxones & M-0 & M-1 & M-2 & Total \\
\hline \multirow{11}{*}{ Bosque mixto } & Quercus sp. caducifolio & 68 & 134 & 283 & 485 \\
\hline & Corylus avellana & 10 & 44 & 46 & 100 \\
\hline & Castanea sativa & 2 & 13 & 25 & 40 \\
\hline & Prunus sp. & 3 & 3 & 25 & 31 \\
\hline & Rosaceae/Maloideae & 2 & 7 & 10 & 19 \\
\hline & Quercus/Castanea & - & 6 & 3 & 9 \\
\hline & Juglans regia & - & - & 7 & 7 \\
\hline & Quercus sp. perennifolio & 3 & - & - & 3 \\
\hline & Quercus sp. & - & 1 & 1 & 2 \\
\hline & Fraxinus sp. & - & 1 & 1 & 2 \\
\hline & Frangula alnus & - & 1 & - & 1 \\
\hline \multirow{2}{*}{ Bosque de ribera } & Salix/Populus & 1 & 2 & 6 & 9 \\
\hline & Sambucus sp. & 1 & - & - & 1 \\
\hline \multirow{4}{*}{ Matorral } & Fabaceae & 5 & 10 & 21 & 36 \\
\hline & Erica sp. & 3 & 9 & 18 & 30 \\
\hline & Juniperus sp. & - & 1 & - & 1 \\
\hline & Erica arborea/australis & - & - & 1 & 1 \\
\hline \multirow[t]{2}{*}{ Otros } & Corteza & 1 & 2 & 21 & 24 \\
\hline & Total & 100 & 238 & 468 & 806 \\
\hline
\end{tabular}

presenten un aspecto vítreo (fig. 5b), provocado por la fusión de los diferentes elementos anatómicos de la madera, que homogeneizan su estructura, pudiendo llegar incluso a provocar la fusión total de los tejidos y a impedir la identificación anatómica por la pérdida de características como puntuaciones, perforaciones, engrosamientos espiralados, células de parénquima, etc. (Théry-Parisot 2001, McParland et al. 2010). El registro de la presencia de grietas radiales y de vitrificación en los carbones puede proporcionar información sobre las condiciones de combustión de la madera e incluso sobre el estado de esta de forma previa a la combustión.

La fragmentación es uno de los efectos de la combustión y su intensidad es diferente en función de las características anatómicas de la madera, pero también puede estar relacionada con procesos deposicionales y postdeposicionales (Lancelotti et al. 2010). Cada fragmento analizado fue medido en su lado más largo, con el objetivo de registrar el índice de fragmentación y para realizar una valoración de los procesos de formación del contexto arqueológico en el que se localizan. Los grados de fragmentación establecidos son de 0,3-1 $\mathrm{cm}$ muestra muy fragmentada, de más de 1 a $3 \mathrm{~cm}$ fragmentada, de más de 3 a 10 moderadamente fragmentada y de más de $10 \mathrm{~cm}$ poco fragmentada (Martín-Seijo 2013). Para registrar los procesos de movilización a los que se pueden ver sometidos los carbones registramos también el tipo de aristas que presentaban, estableciendo dos categorías: angulosas o redondeadas (Rodríguez-Ariza 1993, Martín-Seijo 2013).

\section{RESULTADOS}

El análisis antracológico ha permitido identificar 17 taxones arbóreos y arbustivos (tab. 2): Quercus sp. 


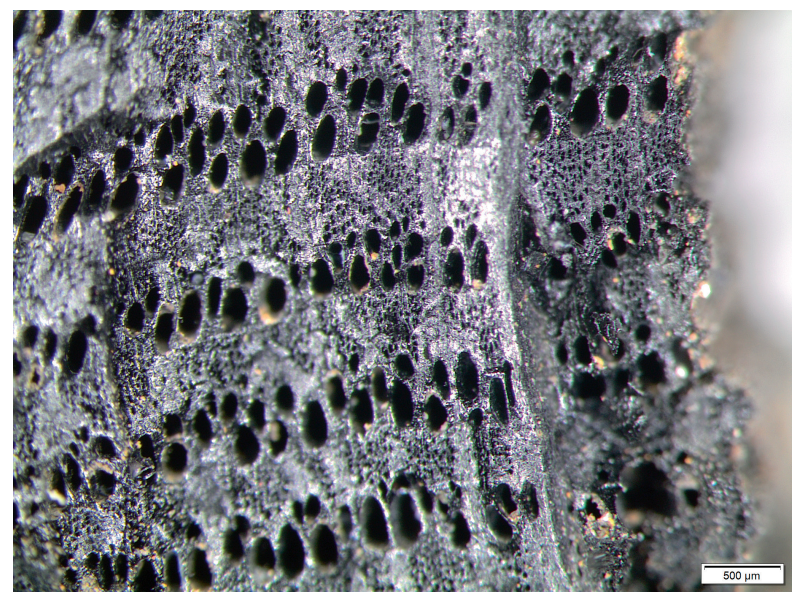

a

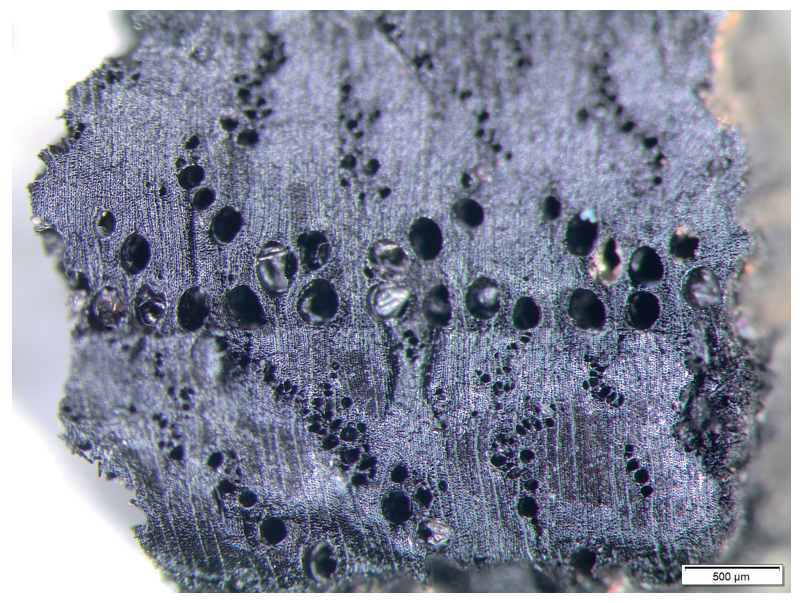

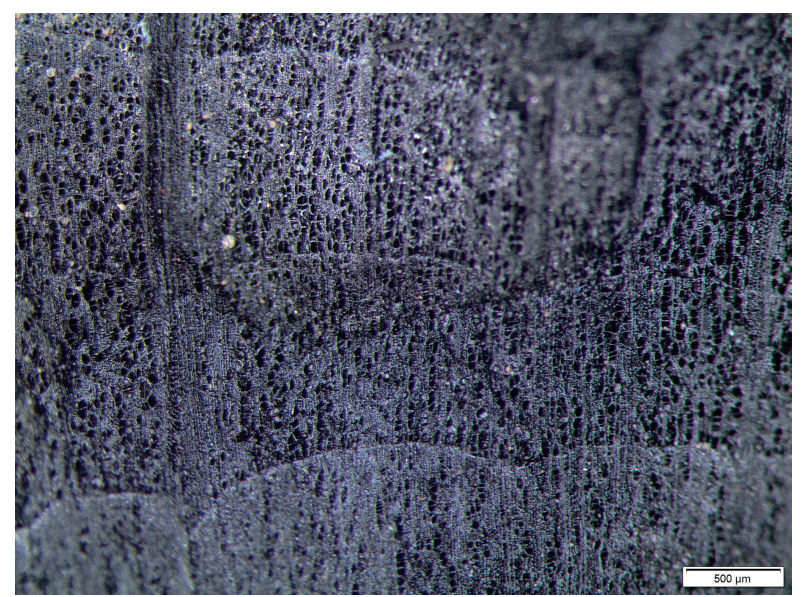

b

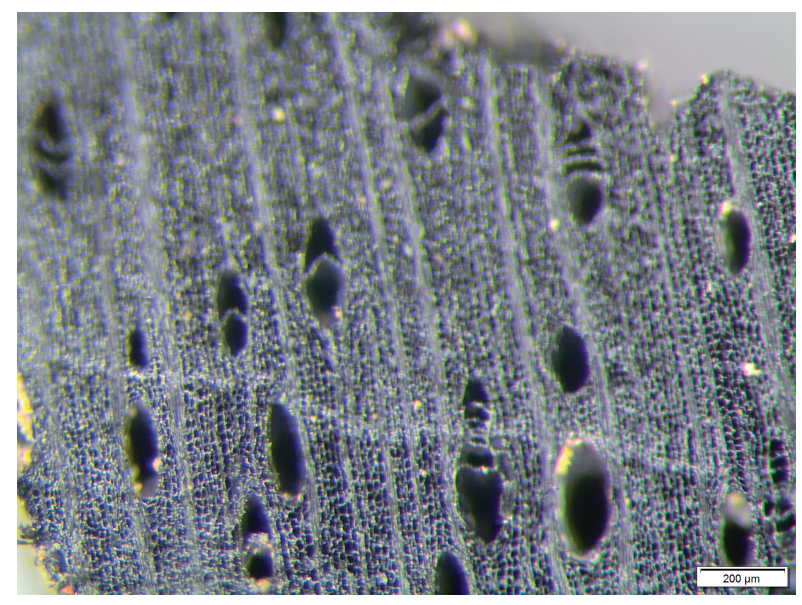

d

Fig. 6. Planos transversales de varios de los taxones identificados durante el análisis antracológico: a) Quercus sp. caducifolio, b) Corylus avellana, c) Castanea sativa, d) Juglans regia.

caducifolio, Corylus avellana, Castanea sativa, Fabaceae, Erica sp., Prunus sp., Rosaceae/Maloideae, Salix/Populus, Quercus/Castanea, Fraxinus sp., Quercus sp., Quercus sp. perennifolio, Sambucus sp., Frangula alnus, Juniperus sp., Juglans regia y Erica arborea/australis. Se han identificado también algunos fragmentos de corteza que no ha sido posible asignar a ningún taxón.

Los datos dendrológicos nos han permitido caracterizar los recursos leñosos consumidos más allá de la identificación taxonómica. En el $80,8 \%$ de los fragmentos pudo ser registrado el tipo de curvatura, predominando en el conjunto los fragmentos con curvatura moderada, seguida de aquellos con curvatura fuerte (tab. 3). En los taxones consumidos más habitualmente como Quercus sp. caducifolio, Corylus avellana,
Castanea sativa o Prunus sp. hay un claro predominio de los fragmentos de curvatura moderada, mientras que en taxones arbustivos como Fabaceae y Erica sp. hay un predominio de los fragmentos con curvatura fuerte (tab. 3).

La parte de la planta pudo ser identificada a partir de sus características morfológicas y anatómicas en 47 fragmentos, el 5,9\% de los casos (tab. 4). En 4 de los carbones las ramas conservaban la médula y la corteza, por lo que pudo ser recontada la edad en el momento de la muerte, 2 anillos anuales en un fragmento de Rosaceae/Maloideae y en dos fragmentos de Quercus sp. caducifolio, y 3 anillos anuales en un fragmento de Quercus sp. caducifolio.

En el conjunto de la muestra se han identificado diferentes tipos de alteraciones anatómicas o 
Tabla 3. Datos cualitativos de la curvatura en el anillo de los diferentes taxones identificados en Pambre: F: fuerte, F-M: fuerte-moderada, M: moderada, M-D: moderada-débil, D: débil, I: indeterminado.

\begin{tabular}{|c|c|c|c|c|c|c|}
\hline Taxones/Curvatura & $\mathrm{F}$ & F-M & M & M-D & $\mathrm{D}$ & I \\
\hline Quercus sp. caducifolio & 55 & 5 & 226 & 44 & 40 & 115 \\
\hline Corylus avellana & 13 & - & 80 & - & 2 & 5 \\
\hline Castanea sativa & 3 & - & 25 & 4 & 5 & 3 \\
\hline Fabaceae & 28 & 1 & 6 & - & & 1 \\
\hline Prunus sp. & 2 & - & 18 & 2 & 7 & 2 \\
\hline Erica sp. & 20 & - & 5 & - & - & 3 \\
\hline Rosaceae/Maloideae & 4 & - & - & - & 1 & 14 \\
\hline Quercus/Castanea & 3 & - & 2 & - & - & 4 \\
\hline Salix/Populus & 2 & - & 3 & - & 1 & 3 \\
\hline Juglans regia & - & - & 2 & - & 5 & - \\
\hline Quercus sp. perennifolio & - & - & 1 & - & - & 2 \\
\hline Fraxinus sp. & - & - & 2 & - & - & \\
\hline Quercus sp. & - & - & & - & 1 & 1 \\
\hline Frangula alnus & - & - & 1 & - & - & - \\
\hline Sambucus sp. & - & - & 1 & - & - & - \\
\hline Juniperus sp. & - & - & - & - & - & 1 \\
\hline Erica arborea/australis & - & - & - & - & - & 1 \\
\hline Total & 130 & 6 & 372 & 50 & 62 & 155 \\
\hline
\end{tabular}

morfológicas (tab. 5). Se han registrado alteraciones del ritmo de crecimiento (narrow rings) en el 5,6\% de los fragmentos de Quercus sp. caducifolio, cicatrices en el 26,3\% de los fragmentos de Rosaceae/Maloideae, y compartimentalización en el 16\% de Corylus avellana y 11,1\% de Salix/Populus. La presencia de evidencias de madera degradada es muy puntual y afecta solo a algunos fragmentos de Quercus sp. caducifolio, Corylus avellana y corteza. Las alteraciones más frecuentes son aquellas relacionadas con el proceso de combustión como la vitrificación $(25,5 \%)$ y las grietas radiales $(23,7 \%)$, afectando en mayor proporción a taxones como Fabaceae o Erica sp. La presencia de fragmentos con aristas redondeadas que se podrían relacionar con procesos de movilización es muy puntual y afecta solo al 3\% de los fragmentos analizados.

El tamaño de los fragmentos analizados oscila entre 0,2 y $2 \mathrm{~cm}$ (tab. 6). Predominan los fragmentos con tamaños comprendidos entre 0,5 y $1 \mathrm{~cm}$, seguidos de aquellos con tamaños de 0,2 a 0,5 cm. La muestra está muy fragmentada.

\section{DISCUSIÓN}

Los datos obtenidos a partir del análisis antracológico en el castillo de Pambre proporcionan información sobre la leña consumida por sus ocupantes entre los siglos XV y XVII. Establecer el uso de esta leña es complejo, no obstante, al estar asociados estos carbones a restos óseos y a ictiofauna, podemos plantear la posibilidad de que estuvieran relacionados con el procesado de alimentos; aunque no podemos descartar completamente que hubieran sido utilizados para la iluminación y calefacción de alguna de las estancias del palacio o de alguna de las construcciones del recinto fortificado, y que fueran posteriormente mezclados con residuos de comida. 
Tabla 4. Parte de la planta identificada: LI: leño indeterminado; RA: rama; N: nudo, C: corteza.

\begin{tabular}{|c|c|c|c|c|}
\hline Taxones/Parte de la planta & LI & RA & $\mathrm{N}$ & $\mathrm{C}$ \\
\hline Quercus sp. caducifolio & 470 & 11 & 4 & - \\
\hline Corylus avellana & 99 & - & 1 & - \\
\hline Castanea sativa & 40 & - & - & - \\
\hline Fabaceae & 30 & 6 & - & - \\
\hline Prunus sp. & 40 & - & - & - \\
\hline Erica sp. & 23 & 4 & 3 & - \\
\hline Rosaceae/Maloideae & 18 & 1 & - & - \\
\hline Quercus/Castanea & 9 & - & - & - \\
\hline Salix/Populus & 9 & - & - & - \\
\hline Juglans regia & 7 & - & - & - \\
\hline Quercus sp. perennifolio & 3 & - & - & - \\
\hline Fraxinus sp. & 2 & - & - & - \\
\hline Quercus sp. & 2 & - & - & 一 \\
\hline Frangula alnus & 1 & - & - & - \\
\hline Sambucus sp. & 1 & - & - & - \\
\hline Juniperus sp. & 1 & - & - & - \\
\hline Erica arborea/australis & 1 & - & - & - \\
\hline Indeterminado & - & - & - & 24 \\
\hline Total & 755 & 22 & 8 & 24 \\
\hline
\end{tabular}

La escasa presencia de carbones con aristas redondeadas en la muestra estudiada indicaría que no se vieron afectados por procesos de movilización más que de forma puntual, a pesar de que el tamaño de los fragmentos analizados indica que la muestra se encontraba muy fragmentada.

La identificación de 17 taxones en el conjunto de las muestras analizadas indica una explotación diversificada del entorno para la obtención de leña, no solo por la variedad taxonómica sino por las diferentes formaciones vegetales en las que estas comunidades se aprovisionaban de leña. El bosque mixto de planocaducifolios era la formación arbórea en la que obtenían la mayor parte del combustible y la mayor variedad de taxones: Quercus sp. caducifolio, Quercus sp., Castanea sativa, Fraxinus sp., Juglans regia y Quercus sp. perennifolio. También se explotaban probablemente las áreas de claro existentes en su interior o su orla arbustiva, tal y como indica la presencia de Corylus avellana, Prunus sp., Rosaceae/Maloideae y Frangula alnus. Las formaciones de ribera (Salix/Populus, Sambucus sp.) y las de matorral (Fabaceae, Erica sp., Erica arborea/australis y Juniperus sp.) fueron también utilizadas.

No se documenta el consumo de leña afectada por degradación biológica, solo se ha identificado la presencia esporádica de la acción de insectos xilófagos sobre Corylus avellana. Este hecho podría indicar que no existía una recolección de madera muerta o un almacenaje de la leña durante largos períodos, o que, si este se producía, se realizaba en condiciones que no favorecían la degradación de la madera. Las condiciones que favorecen el crecimiento de microorganismos son una elevada humedad de aproximadamente el 40-80\%, temperaturas de entre $1-50^{\circ} \mathrm{C}$ y nivel de $\mathrm{pH}$ alrededor de 6 (Blanchette 2000, Schmidt 2006). 
Tabla 5. Alteraciones relacionadas con la vida de la planta. AC: anillos estrechos relacionados con alteraciones de crecimiento, CI: cicatrices, CO: compartimentalización; HI: hifas, XI: galerías de xilófagos, GR: grietas radiales, GT: grietas tangenciales, VT: vitrificación, ER: erosión.

\begin{tabular}{|c|c|c|c|c|c|c|c|c|c|}
\hline Taxones/Parte de la planta & $\mathrm{AC}$ & $\mathrm{CI}$ & $\mathrm{CO}$ & $\mathrm{HI}$ & $\mathrm{XI}$ & VT & GR & GT & ER \\
\hline Quercus sp. caducifolio & 27 & - & - & 2 & - & 146 & 121 & - & 5 \\
\hline Corylus avellana & - & - & 16 & - & 2 & 10 & 17 & - & 7 \\
\hline Castanea sativa & - & - & - & - & - & 3 & 1 & - & 1 \\
\hline Fabaceae & - & - & - & - & - & 19 & 24 & - & \\
\hline Prunus sp. & - & - & - & - & - & 6 & 3 & - & 2 \\
\hline Erica sp. & - & - & - & - & - & 13 & 19 & 1 & 1 \\
\hline Rosaceae/Maloideae & - & 5 & - & - & - & 4 & - & - & 2 \\
\hline Quercus/Castanea & - & - & - & - & - & - & 2 & - & - \\
\hline Salix/Populus & - & - & 1 & - & - & - & - & - & 1 \\
\hline Juglans regia & - & - & - & - & - & 1 & 1 & - & 2 \\
\hline Quercus sp. perennifolio & - & - & - & - & - & 1 & 1 & - & 1 \\
\hline Fraxinus sp. & - & - & - & - & - & - & - & - & - \\
\hline Quercus sp. & - & - & - & - & - & - & - & - & 一 \\
\hline Frangula alnus & - & - & - & - & - & - & - & - & - \\
\hline Sambucus sp. & - & - & - & - & - & 1 & - & - & 一 \\
\hline Juniperus sp. & - & - & - & - & - & - & - & - & 1 \\
\hline Erica arborea/australis & - & - & - & - & - & 1 & 1 & - & - \\
\hline Indeterminado & - & - & - & 1 & - & 1 & 1 & - & 1 \\
\hline Total & 27 & 5 & 17 & 3 & 2 & 206 & 191 & 1 & 24 \\
\hline
\end{tabular}

El registro de las alteraciones que registran las condiciones de vida de las plantas aporta datos significativos en el caso de Quercus sp. caducifolio, Rosaceae/ Maloideae, Corylus avellana y Salix/Populus. En el primer caso, se documenta en un pequeño porcentaje de fragmentos la supresión del crecimiento del anillo anual durante el leño final, que podría relacionarse con la existencia de prácticas de poda como el trasmocho, el desmocho o la entresaca, relativamente habituales en esta época para mejorar la productividad de determinadas especies (Aragón-Ruano 2001, 2009), aunque no sería descartable que también que se asociaran a períodos de estrés hídrico o condiciones de crecimiento desfavorables (Schweingruber 2007, Schweingruber y Börner 2018). Teniendo en cuenta la limitación de esta evidencia, y la sucesión de períodos fríos durante la Pequeña Edad del Hielo, no podemos descartar ninguna de estas posibilidades, aunque sí es significativo que esta alteración solo se documente sobre Quercus sp. caducifolio. Este hecho podría apuntar a la existencia de poda sobre este árbol.

Por otra parte, la presencia de cicatrices sobre Rosaceae/Maloideae en un porcentaje elevado podría indicar que un individuo, o individuos de este grupo de árboles se vio afectado por algún factor que dañó el cambium: incendio, acción biológica o antrópica (Schweingruber 2007, Schweingruber y Börner 2018). Finalmente, la presencia de compartimentalización en fragmentos de Corylus avellana y Salix/Populus podría indicar que existían en el entorno del sitio un individuo o individuos de estos taxones que estaba afectado por algún tipo de microorganismos. 
Tabla 6. Tamaño de los fragmentos de carbón analizados

\begin{tabular}{|c|c|c|c|c|}
\hline Taxones/Tamaño (cm) & $0,2-0,5$ & $>0,5-1$ & $>1-1,5$ & $>1,5-2$ \\
\hline Quercus sp. caducifolio & 168 & 279 & 35 & 3 \\
\hline Corylus avellana & 31 & 58 & 7 & 4 \\
\hline Castanea sativa & 16 & 19 & 4 & 1 \\
\hline Fabaceae & 11 & 21 & 3 & 1 \\
\hline Prunus sp. & 13 & 15 & 3 & - \\
\hline Erica sp. & 16 & 9 & 5 & - \\
\hline Rosaceae/Maloideae & 8 & 10 & - & 1 \\
\hline Quercus/Castanea & 7 & 2 & - & - \\
\hline Salix/Populus & 5 & 4 & - & - \\
\hline Juglans regia & 3 & 3 & 1 & - \\
\hline Quercus sp. perennifolio & - & 3 & - & - \\
\hline Fraxinus sp. & 2 & - & - & - \\
\hline Quercus sp. & 1 & 1 & - & - \\
\hline Frangula alnus & 1 & - & - & - \\
\hline Sambucus sp. & & - & 1 & - \\
\hline Juniperus sp. & 1 & - & - & - \\
\hline Erica arborea/australis & 1 & - & - & - \\
\hline Indeterminado & 8 & 16 & 1 & - \\
\hline Total & 292 & 440 & 60 & 10 \\
\hline
\end{tabular}

Con respecto a los taxones identificados como combustible, destaca en primer lugar el predominio de la madera obtenida de árboles y arbustos, frente a la escasa importancia que tienen en el conjunto los taxones arbustivos asociados a las formaciones de matorral (Fabaceae, Erica sp., Erica arborea/australis, Juniperus sp.). En general predomina la leña de calibre medio a pequeño, tanto en los taxones predominantes (Quercus sp. caducifolio, Corylus avellana, Castanea sativa, Prunus sp.) como en aquellos menos frecuentes y de porte arbustivo (Fabaceae, Erica spp.). Se ha identificado el uso de ramas de Quercus sp. caducifolio, Fabaceae, Erica sp. y Rosaceae/Maloideae, en algún caso de solo 2-3 años. La presencia de curl wood ha permitido identificar también la presencia de nudos, pequeños brotes durmientes de las ramas, también en fragmentos de Quercus sp. caducifolio, Corylus avellana y Erica sp. El uso predominante de leña obtenida de ramas podría estar relacionada con las restricciones existentes desde época bajomedieval destinadas a proteger a los árboles y el bosque (Carlé 1976), o con el uso como combustible de madera obtenida durante la poda o a partir del desmoche, el trasmoche o el clareo.

Las alteraciones relacionadas con el proceso de combustión, como la vitrificación y las grietas radiales, afectan especialmente a taxones como Fabaceae o Erica spp. Ambos taxones son proclives a presentar este tipo de alteraciones quizá por su comportamiento durante la combustión, en el caso de Erica spp. las grietas son cortas y numerosas, y podrían estar relacionadas con el consumo de leña que conserva un cierto grado de humedad como han propuesto a partir de experimentos sobre coníferas Théry-Parisot y Henry (2012). Este dato nos permitiría plantear que las arbustivas pudieron haber sido consumidas en verde o húmedas, lo que indicaría un aprovisionamiento próximo al momento de 
uso como combustible, sin almacenaje previo que permitiera esa pérdida de humedad. Las fuentes señalan la presencia de tojales y codesales lindantes a las tierras de cultivo y que entre otros usos también eran aprovechados para forraje y para el abono de la tierra (Ríos Rodríguez 1997), y puede que se privilegiara este uso frente a su consumo como combustibles debido a la poca importancia que tienen en el conjunto estos taxones.

Es significativa también la importancia, tanto a nivel porcentual como por ubicuidad, de las muestras de taxones frutales silvestres y cultivados, tanto aquellos de porte arbóreo como los de porte arbustivo (Corylus avellana, Castanea sativa, Prunus sp., Rosaceae/Maloideae, Sambucus sp. y Juglans regia). A pesar de que algunas de estas especies pueden aparecer asociadas a los bosques mixtos de planocaducifolios (Corylus avellana, Sambucus sp.), hay otros que podemos agrupar dentro de la categoría de árboles fuera del bosque, tanto por su vinculación a prácticas de arboricultura como a su relevancia económica para estas comunidades: Castanea sativa, Prunus sp., Rosaceae/Maloideae y Juglans regia. Los árboles fuera del bosque serían aquellos que crecen próximos a las áreas de habitación y fuera de las masas boscosas. Este término es un neologismo que se define en relación al bosque y por defecto, agrupando a los árboles localizados en tierras que no pertenecen a la categoría de bosques o tierras forestales, ni a otras áreas boscosas, que pueden ser espontáneas o plantadas, generalmente cultivadas y conservadas (Bellefontaine et al. 2002: 2, Kleinn 2002: 74, 2000: 4). Se encuentran en tierras agrícolas, con construcciones, en los márgenes de los ríos o arroyos dentro del paisaje agrícola, o en tierras naturales en las que la cobertura arbórea es tan escasa que la vegetación no cumple con la definición de bosque (Kleinn 2002: 74). Esta categoría de clasificación es especialmente interesante para este momento en el que las fuentes distinguen entre lo culto y lo inculto, estando el bosque -silvae, montae, pascuae - en esta última categoría, mientras que este otro tipo de formaciones forestales eran manejadas y cultivadas como los soutos, las devesas, los pomares, los avellanales, etc. (Ríos Rodríguez 1997).

El castaño (Castanea sativa) es junto con la vid uno de los principales cultivos en los terrenos baldíos desde la Edad Media (López-Sabatel 2009). La expansión a gran escala de Castanea está documentada en diversos análisis polínicos del noroeste peninsular desde la Edad Media (Santos et al. 2000, López-Sáez et al. 2003), en una tendencia que tiene paralelos en otras áreas europeas (Conedera et al. 2004). El castaño estaba integrado en las tierras cultivadas, mezclado con todo tipo de cultivos, apareciendo referencias a este árbol en la documentación, con una presencia predominante en el paisaje agrario (Ríos Rodríguez 1997). Las castañas constituían un aporte nutritivo básico y el castaño era incluso objeto de sucesión testamentaria, su madera se utilizaba para la construcción de aperos, muebles domésticos y trabajos artesanales, y su leña se consumía en los hogares, hornos y cocinas (López-Sabatel 2009). De hecho, ciertos pagos en especie referidos en las fuentes documentales se refieren directamente a tareas de los foreros relacionadas con la arboricultura, como la poda y el injerto del castaño (López-Sabatel 2011).

Frente al castaño y a otros árboles frutales como nogales, higueras, manzanos, perales, cerezos, etc., el roble tiene en las referencias documentales una importancia secundaria (López-Sabatel 2009) a pesar de ser el combustible predominante en todo tipo de asentamientos durante la época medieval y moderna (tab. 6). La presencia de madera de frutales, si tenemos además en cuenta el calibre de la leña consumida, que sería de mediano a pequeño calibre, podría estar relacionada con el aprovechamiento de los residuos de poda como combustible. En el caso de determinados taxones, como los manzanos y los perales (Rosaceae/Maloideae) o los cerezos (Prunus sp.), probablemente procedían de árboles y arbustos situados en el entorno inmediato al lugar de habitación (cortiñas), ya que sus frutos no podían ser almacenados y tenían que ser consumidos de forma casi inmediata (López-Sabatel 2009). La presencia de madera de Prunus sp. es también relevante. A pesar de que no es excesivamente significativa a nivel porcentual, es un taxon que suele aparecer de forma recurrente en el registro antracológico asociado a contextos de cronología medieval y moderna en el norte peninsular (Allué 2003, Teira et al. 2011, Ruiz-Alonso et al. 2012). También se han documentado de forma recurrente conjuntos de semillas de Prunus avium/cerasus en contextos de época medieval del noroeste peninsular (Teira 2013, 2015; Peña-Chocarro et al. 2019).

Con respecto a la pregunta de quién realizó el aprovisionamiento de la leña consumida en el interior del castillo de Pambre, las fuentes textuales nos indican que las prestaciones en trabajo derivadas de la expresión de señoría, que incluían la recolección de leña en el monte, además de otras tareas agrícolas o exigencias de trabajos sobre infraestructuras, y que eran habituales desde el siglo $\mathrm{X}$ y hasta la segunda mitad del siglo XIII, fueron cada vez menos frecuentes, $y$ a mediados del siglo XIV prácticamente no se encuentra referencia a las mismas (López-Sabatel 2011). La ausencia de 
Tabla 7. Comparación de los datos antracológicos obtenidos en contextos medievales y modernos del noroeste ibérico.

\begin{tabular}{|c|c|c|c|c|c|c|c|c|}
\hline & \multicolumn{2}{|c|}{ Cova Eirós } & \multicolumn{2}{|c|}{ Peñaferruz } & \multicolumn{2}{|c|}{ Pambre } & \multicolumn{2}{|c|}{ Mourela } \\
\hline Tipo de sitio & \multicolumn{2}{|c|}{ Cueva } & \multicolumn{2}{|c|}{ Castillo } & \multicolumn{2}{|c|}{ Castillo } & \multicolumn{2}{|c|}{ Rural } \\
\hline Cronología & \multicolumn{2}{|c|}{ s. X-XI } & \multicolumn{2}{|c|}{ s. IX-XIII } & \multicolumn{2}{|c|}{ s. XV-XVII } & \multicolumn{2}{|c|}{ s. XIV-XVII } \\
\hline Taxones & $\mathrm{N}^{\mathrm{o}}$ & $\%$ & $\mathrm{~N}^{\mathrm{o}}$ & $\%$ & $\mathrm{~N}^{\mathrm{o}}$ & $\%$ & $\mathrm{~N}^{\mathrm{o}}$ & $\%$ \\
\hline Quercus sp. caducifolio & 15 & 14,2 & 181 & 60,0 & 485 & 60,2 & 887 & 76,9 \\
\hline Corylus avellana & 2 & 1,9 & 26 & 8,6 & 100 & 12,5 & - & - \\
\hline Castanea sativa (y cf.) & 3 & 2,8 & 13 & 4,3 & 40 & 5,0 & - & - \\
\hline Fabaceae (y cf.) & 4 & 3,8 & 17 & 5,6 & 36 & 4,5 & 23 & 2,0 \\
\hline Prunus sp. & 1 & 0,9 & 2 & 0,7 & 31 & 3,8 & - & - \\
\hline Erica sp. (y cf.) & - & - & - & - & 30 & 3,7 & 220 & 19,1 \\
\hline Rosaceae/Maloideae (y cf.) & 15 & 14,2 & 10 & 3,3 & 19 & 2,4 & 18 & 1,5 \\
\hline Salix/Populus & 29 & 27,6 & - & - & 9 & 1,1 & - & - \\
\hline Quercus/Castanea & - & - & - & - & 9 & 1,1 & - & - \\
\hline Juglans regia & - & - & - & - & 7 & 0,9 & - & - \\
\hline Quercus sp. perennifolio & - & - & 1 & 0,3 & 3 & 0,4 & - & - \\
\hline Fraxinus sp. (y cf.) & 6 & 5,7 & - & - & 2 & 0,2 & - & - \\
\hline Quercus sp. & - & - & - & - & 2 & 0,2 & - & - \\
\hline Sambucus sp. & - & - & 1 & 0,3 & 1 & 0,1 & - & - \\
\hline Frangula alnus & - & - & - & - & 1 & 0,1 & - & - \\
\hline Juniperus sp. & - & - & - & - & 1 & 0,1 & - & - \\
\hline Erica arborea/australis & - & - & - & - & 1 & 0,1 & - & - \\
\hline Ulmus sp. & 7 & 6,6 & 1 & 0,3 & - & - & - & - \\
\hline Arbutus unedo & 1 & 0,9 & 3 & 1,0 & 一 & - & - & 一 \\
\hline Betula sp. & 22 & 20,9 & 1 & 0,3 & - & - & 2 & 0,2 \\
\hline Fagus sylvatica & - & - & 14 & 4,6 & - & - & - & - \\
\hline Laurus nobilis & - & - & 1 & 0,3 & - & - & - & - \\
\hline Ilex aquifolium & - & - & - & - & 一 & - & 4 & 0,3 \\
\hline Corteza & - & - & - & - & 24 & 3,0 & - & - \\
\hline Indeterminable & - & - & 11 & 3,6 & 一 & - & - & - \\
\hline Total & 105 & 100 & 302 & 100 & 806 & 100 & 1154 & 100 \\
\hline
\end{tabular}

galerías de insectos xilófagos indicaría que la leña probablemente no era almacenada durante mucho tiempo, mientras que el predominio de la madera de mediano y pequeño calibre obtenida del bosque de robles, así como de los árboles fuera del bosque, podría estar relacionado con las restricciones destinadas a proteger el bosque y determinados árboles (Carlé 1976), así como con el aprovechamiento de los restos de la poda como 
leña. La escasa importancia de la leña obtenida de las especies de matorral sí podría indicar que no se aprovisionan en los espacios comunales de monte más que de forma puntual, o que los matorrales eran utilizados de forma prioritaria para la obtención de forraje para el ganado y abono para los campos de cultivo (Ríos Rodríguez 1997).

La legislación proteccionista sobre bosques y árboles (Carlé 1976), así como el retroceso del bosque, que es acusado en este momento tal y como está documentado en diferentes secuencias palinológicas del noroeste peninsular (Martínez-Cortizas et al. 2000, Santos et al. 2000, Desprat et al. 2003, López-Sáez et al. 2003, Martínez-Cortizas et al. 2005, López-Merino et al. 2014, Silva-Sánchez et al. 2014), puede ser el motivo de que haya una gran diversificación en el aprovisionamiento, incluyendo árboles frutales, y una preferencia por leña de mediano a pequeño calibre, obtenido probablemente de ramas de mediano y pequeño tamaño, frente a la preferencia por madera de gran calibre que implicaría abatir árboles. El uso de las ramas, incluyendo en algunos casos la posibilidad de la existencia de prácticas como el desmoche, el trasmoche o el clareo, permitiría asegurar una renovación cíclica de los recursos, o el aprovechamiento de los residuos de la poda en el caso de los frutales. El uso preferente de leña de pequeño calibre tendría probablemente implicaciones en lo que respecta a la organización y estacionalidad de este aprovisionamiento, así como incluso respecto al tipo de herramientas utilizadas, fundamentalmente podones frente al uso de hachas.

Si comparamos los datos obtenidos en Pambre con otros contextos de época medieval y moderna del noroeste peninsular, podemos observar cómo durante todo este período parece existir una preferencia por la madera obtenida a partir del roble y otras especies caducifolias de Quercus y cómo la importancia de lo que podemos clasificar como árboles fuera del bosque se mantiene durante todo el período (tab. 7). El uso de la madera de Castanea como leña se documenta tanto en la ocupación en cueva de Cova Eirós (Teira et al. 2011) como en el castillo de Curiel en Peñaferruz (Allué 2003). A pesar de que en este último contexto también se documenta una cierta variabilidad taxonómica, la diversidad de taxones documentada en Pambre destaca sobre el conjunto. Esto puede estar condicionado por diferentes factores, por una parte con la propia organización de la recolección de la leña, pero por otra parte tampoco sería descartable que el tipo de contexto en el que las muestras fueron recuperadas, que acumularía combustiones realizadas durante un período de tiempo indeterminado, haya contribuido a esta diversidad. Las diferencias son acusadas cuando comparamos los datos de Pambre con los de A Mourela (Martín-Seijo et al. 2009, 2010), un asentamiento rural relacionado con una explotación pastoril de un área de monte. Esta diferencia nos llevaría a plantear la posibilidad de que existiera un acceso diferencial a los recursos forestales en uno y otro caso, más allá de aquellas diferencias que puedan derivar de los recursos disponibles en el entorno de cada asentamiento y del carácter permanente o temporal de la ocupación. Estas diferencias entre ambos podrían indicar que en el castillo existía un acceso a un mayor número de formaciones arbóreas y arbustivas en las que podía ser recolectada leña, frente a A Mourela donde únicamente se documenta el consumo de leña del bosque de caducifolios, fundamentalmente roble, y de especies de matorral.

\section{CONCLUSIONES}

El análisis antracológico de las muestras de carbón recuperadas en Pambre nos ha permitido obtener información directa sobre el tipo de leña utilizado en este castillo entre los siglos XV y XVII. Este análisis contribuye a aumentar el conocimiento que hasta el momento teníamos sobre el uso de los recursos vegetales durante el final de la Edad Media y el inicio de la Edad Moderna, tanto a partir del registro arqueobotánico como a partir de las fuentes escritas. Estos resultados son especialmente significativos porque los únicos datos de estas cronologías provenían de un asentamiento rural relacionado con actividades pastoriles, mientras que en el caso de Pambre los carbones analizados son probablemente los residuos de la leña quemada para procesar alimentos, o para la calefacción e iluminación de las estancias.

Los resultados obtenidos permiten documentar un aprovechamiento de los recursos leñosos mucho más complejo que lo registrado en las fuentes, y que lo documentado en otros sitios arqueológicos con cronologías similares. Se documenta una gran diversidad taxonómica en la que la mayor parte de la leña fue probablemente obtenida de las formaciones de planocaducifolios y de los árboles fuera del bosque, mientras que la importancia de las especies obtenidas en las formaciones de matorral es poco significativa. La principal madera consumida como leña es el roble (Quercus sp. caducifolio), seguido del avellano (Corylus avellana) y del castaño (Castanea sativa). En todos los casos predomina la madera de pequeño a mediano calibre 
obtenida a partir de especies que producen frutos comestibles, lo que podría estar indicando un aprovechamiento de los residuos de la poda. No se documentan evidencias de consumo de leña muerta o de almacenaje en condiciones favorables para el ataque de microorganismos. La presencia de grietas cortas y numerosas en algunos de los fragmentos de carbón podría apuntar incluso al consumo de madera verde o húmeda. El escaso peso que en el conjunto tienen taxones como Fabaceae o Erica spp. podría estar indicando que probablemente estas plantas se están utilizando con otros fines.

\section{Agradecimientos}

La investigación realizada por María Martín-Seijo ha sido financiada por una beca postdoctoral Plan I2C mod. B con el proyecto "MATERIAL-Materiality and Material Culture: Wood and Other Plant-based Materials in Archaeological Contexts". La intervención arqueológica realizada en el castillo de Pambre fue dirigida por Santiago Vázquez Collazo y financiada por la Secretaría Xeral para o Turismo de la Consellería de Presidencia de la Xunta de Galicia. Los autores quieren expresar su agradecimiento a Carlos Fernández Rodríguez de la Universidad de León, que se encargó de la recogida y procesado de las muestras. El análisis antracológico se llevó a cabo en el Laboratorio de Arqueobotánica del Grupo de Estudos do Noroeste Ibérico-Arqueología, Antigüidade e Territorio (GEPN-AAT) de la Universidade de Santiago de Compostela.

\section{BIBLIOGRAFÍA}

Allué, E. (2003): “Antracología”, en J.A. Gutiérrez (ed.). Peñaferruz (Gijón): el castillo de Curiel y su territorio: 375-381. Gijón, Ayuntamiento de Gijón/ Vtp Editorial.

Amigo, J.; Rodríguez-Guitián, M.A.; Honrado, J.J.P. y Alves, P. (2017): "The Lowlands and Midlands of Northwestern Atlantic Iberia", en J. Loidi (ed.), The Vegetation of the Iberian Peninsula: 191-250. Berlín, Springer. doi: $<$ https://doi.org/10.1007/978-3319-54784-8>.

Aragón-Ruano, Á. (2001): "El bosque guipuzcoano en la Edad Moderna: aprovechamiento, ordenamiento legal y conflictividad". Munibe. Suplemento 14: $1-285$.

Aragón-Ruano, Á. (2009): "Una longeva técnica forestal: los trasmochos o desmochos guiados en
Guipúzcoa durante la Edad Moderna". Espacio, Tiempo y Forma, Serie IV, Historia Moderna 22: 73-105. doi: <https://doi.org/10.5944/etfiv.22.2009.1609>.

Armas Castro, J. (1992): Pontevedra en los siglos XII a XV. Configuración y desarrollo de una villa marinera en la Galicia medieval, Pontevedra. A Coruña, Fundación Barrié de la Maza.

Bellefontaine, R.; Petit, S.; Pain-Orcet, M.; Deleporte, Ph. y Bertault; J.-G. (2002): Los árboles fuera del bosque. Hacia una mejor consideración. Guía FAO Conservación 35. Roma, FAO.

Blanchette, R.A. (2000): "A review of microbial deterioration found in archaeological wood from different environments". International Biodeterioration \& Biodegradation 46 (3): 189-204.

Burri, S. (2010): "Le problème de la mobilité des artisans, pasteurs et usagers de l'incultum en basseProvence centrale (XIIe-XVIe siècle)". Migrations et mobilités au Moyen Âge. Histoire ancienne et médiévale 104: 131-136.

Burri, S. (2018): "Chapter 8: Ancient forest and oldgrowth forest from the perspective of medieval and modern written sources. The case of the 'Bois de la Sainte-Baume' (France, AD late 13th to early 19th centuries)", en S. Paradis-Grenouillet, S. Burri y Ch. Aspe (eds.), Into the woods. Overlapping perspectives on the history of ancient forests: 99-110. Versailles Cedex, Quae.

Carlé, M. C. (1976): "El bosque en la Edad Media (Asturias-León-Castilla)". Cuadernos de Historia de España 59: 297-375.

Carrión, Y. (2005): La vegetación mediterránea y atlántica de la península Ibérica. Nuevas secuencias antracológicas. Servicio de Investigación Prehistórica, 104. Valencia, Diputación Provincial de Valencia.

Cartwright, C.R. (2015): “The principles, procedures and pitfalls in identifying archaeological and historical wood samples". Annals of Botany 116 (1): 1-13. doi: 10.1093/aob/mcv056

Conedera, M.; Krebs, P.; Tinner, W.; Pradella, M. y Torriani, D. (2004): "The cultivation of Castanea sativa (Mill.) in Europe, from its origin to its diffusion on a continental scale". Vegetation History and Archaeobotany 13 (3): 161-179.

Costa, M.; Morla, C. y Saínz, H. (2001): Los bosques ibéricos. Una interpretación geobotánica. Barcelona, Editorial Planeta.

Cubero, C. (2011): "Woody plant resources in the medieval site of l'Esquerda (Roda de Ter, Barcelona, Spain)". Saguntum Extra 11: 171-172. 
Deforce, K.; Boeren, I.; Adriaenssens, S.; Bastiaens, J.; De Keersmaeker, L.; Haneca, K.; Tys, D. y Vandekerkhove, K. (2013): "Selective woodland exploitation for charcoal production. A detailed analysis of charcoal kiln remains (ca. 1300-1900 AD) from Zoersel (northern Belgium)". Journal of Archaeological Science 40 (1): 681-689. doi: <https://doi. org/10.1016/j.jas.2012.07.009>.

Desprat, S.; Goñi, M.F. y Loutre, M.F. (2003): "Revealing climatic variability of the last three millennia in northwestern Iberia using pollen influx data". Earth and Planetary Science Letters 213: 63-78. doi: $10.1016 / \mathrm{S} 0012-821 \mathrm{X}(03) 00292-9$

Devia, C. (2015). "El bosque medieval y el derecho de resistencia de los dominados". Cuadernos Medievales (18): 39-51.

Domínguez-Delmás, M.; van Daalen, S.; AlejanoMonge, R. y Wazny, T. (2018): “Timber resources, transport and woodworking techniques in post-medieval Andalusia (Spain): Insights from dendroarchaeological research on historic roof structures". Journal of Archaeological Science 95: 64-75. doi: $<$ https://doi.org/10.1016/j.jas.2018.05.002>.

Estrella, J. R. y Asla, A. (2010): "El aprovisionamiento de leña en las ciudades de la Baja Edad Media Hispánica”. Estudios de Historia de España 12 (1): 185-200.

Euba, I. (2008): Análisis antracológico de estructuras altimontanas en el Valle de La Vansa-Sierra del Cadi (Alt Urgell) y en el Valle del Matriu (Andorra): explotación de recursos forestales del Neolitico a la época moderna. Tesis doctoral, Universitat Rovira i Virgili, Tarragona. Inédita.

Euba, I. (2009): "La vegetación leñosa y el uso de la madera en tres valles de los Pirineos orientales desde el Neolítico hasta época moderna: análisis antracológico, dendrológico y tafonómico". Pyrenae 40 (2): 7-35.

Euba, I. (2010): "L'exploitation des ressources végétales dans les Pyrénées Orientales durant l'Holocène: analyse anthracologique des structures d'élevage, de fours et de charbonnières dans l'Alt Urgell (Chaîne du Cadí) et la Vallée du Madriu (Andorre)". Quaternaire 21 (3): 305-316. doi: <https:// doi.org/10.4000/quaternaire.5646>.

Euba, I.; Allée, P.; Rouaud, R. y Paradis-Grenouiller, S. (2011): "Metallurgy and forest landscapes from the Gallo-Roman to the Modern Period in Périgord-Limousin (France)". SAGVNTVM Extra 11: 197-198.

Fagan, B. (2008): La pequeña Edad del Hielo. Cómo el clima afectó a la historia de Europa 1300-1850. Barcelona, Gedisa.
Fernández-Cortizo, C. (2016): "La pequeña edad de hielo en Galicia: estado de la cuestión y estudio histórico". Obradoiro de Historia Moderna 25: 9-39.

Fohrer, F.; Toriti, M. y Durand, A. (2017): “Analyse des vermoulures pour la détermination de quelques espèces d'insectes xylophages de la famille des Ptinidae (Coleoptera)". Bulletin de la Société Entomologique de France 122 (2): 133-142.

Fossier, R. (2017): Gente de la Edad Media. Madrid, Ed. Taurus.

Franco-Rubio, G. (2009): "La vivienda en el Antiguo Régimen: de espacio habitable a espacio social". Chronica Nova 35: 63-103.

Gale, R. y Cutler, D. (2000): Plants in Archaeology. Identification manual of vegetative plant materials used in Europe and the southern Mediterranean to c. 1500. Kew, Westbury and Royal Botanic Gardens.

Gomes, R.V. y Gomes, M.V. (2016): A Gestão dos Recursos Florestais Portugueses na Construção $\mathrm{Na}$ val da Idade Moderna. Lisboa, Pórtico.

González-Pérez, C. (1994): A producción tradicional do ferro en Galicia: as grandes ferrerías da provincia de Lugo. Lugo, Servicio de Publicacións-Deputación Provincial de Lugo.

Guitián Rivera, L. (2001): "La destrucción histórica del bosque en Galicia". SEMATA, Ciencias Socials e Humanidades 13: 105-166.

Hather, J.G. (2000): The Identification of the Northern European Woods. A guide for archaeologists and conservators. London, Archetype Publications.

Hazell, Z.; Crosby, V.; Oakey, M. y Marshall, P. (2017): "Archaeological investigation and charcoal analysis of charcoal burning platforms, Barbon, Cumbria, UK". Quaternary International 458: 178-199. doi: $<$ https://doi.org/10.1016/j.quaint.2017.05.025>.

Hickin, N.E. (1963): The insect factor in wood decay. London, Hutchinson \& Co. Ltd.

Huntley, J. (2010): Northern England: A review of wood and charcoal recovered from archaeological excavations in northern England. Londres, English Heritage.

Kleinn, C. (2002): “¿Árboles fuera del bosque?”. Revista Forestal Centroamericana 37: 74.

Knapp, H.; Nelle, O.; y Kirleis, W. (2015): "Charcoal usage in medieval and modern times in the Harz Mountains Area, Central Germany: Wood selection and fast overexploitation of the woodlands". Quaternary International 366: 51-69. doi: <https://doi. org/10.1016/j.quaint.2015.01.053>. 
Lancelotti, C.; Madella, M.; Ajithprasad, P. y Petrie, C.A. (2010): "Temperature, compression and fragmentation: an experimental analysis to assess the impact of taphonomic processes on charcoal preservation". Archaeological and Anthropological Sciences 2 (4): 307-320. doi: <https://doi.org/10.1007/ s12520-010-0046-8>.

Le Goff, J. (2002): La civilización del Occidente medieval. Barcelona, Paidós.

Leonardi, G. (2002): "Le indagini sulle carbonaie di Vobbia, Castello della Pietra: un'esperienza didattica dell'Università di Genova'. Archeologia Postmedievale 6: 145-154.

López-Merino, L.; Cortizas, A. M.; Reher, G. S.; López-Sáez, J. A.; Mighall, T. M. y Bindler, R. (2014): "Reconstructing the impact of human activities in a NW Iberian Roman mining landscape for the last 2500 years". Journal of Archaeological Science 50: 208-218. doi: <https://doi.org/10.1016/j. jas.2014.07.016>.

López-Sabatel, J. A. (2009): "Uso y transformación en espacio agrario del monte y del estrato arbóreo en la Ribeira Sacra durante los siglos XIV y XV". Cuadernos de Estudios Gallegos, 56 (122): 213-233.

López Sabatel, J. A. (2011): "Rentas y exigencias feudales en la tierra de Lemos durante la Baja Edad Media (siglos XIV y XV)". Anuario de Estudios Medievales 41(1): 211-234.

López-Sáez, J.A.; Parcero, C.; Lima, E.; López, P.; Criado, F.; Macías, R.; Martínez-Cortizas, A. y Franco, S. (2003): "Paleopaisajes concretos: polen, suelos y arqueología del yacimiento de As Pontes (Abadín, Lugo)". Trabajos de Prehistoria 60 (1): 139-151.

Ludemann, T.; Michiels, H. G. y Nölken, W. (2004): "Spatial patterns of past wood exploitation, natural wood supply and growth conditions: indications of natural tree species distribution by anthracological studies of charcoal-burning remains". European Journal of Forest Research 123 (4): 283-292. doi: <https://doi.org/10.1007/s10342004-0049-z>

Manrique, E. y Fernández-Cancio, A. (2000): "Extreme climatic events in dendroclimatic reconstructions from Spain". Climatic Change 44: 123-138. doi: <https://doi.org/10.1023/A:1005458018568>.

Marguerie, D. y Hunot, J.Y. (2007): “Charcoal analysis and dendrology: data from archaeological sites in north-western France". Journal of Archaeological Science 34 (9): 1417-1433. doi: <https://doi. org/10.1016/j.jas.2006.10.032>.
Martín-Seijo, M. (2013): A xestión do bosque e do monte dende a Idade do Ferro a época romana no noroeste da península Ibérica: consumo de combustibles e produción de manufacturas en madeira. Tesis Doctoral, Universidad de Santiago de Compostela. Inédita.

Martín-Seijo, M.; Alles, M.J. y Abad, E. (2009): “Análise dos carbóns arqueolóxicos”, en A. Bonilla y R. Fábregas (eds.), Círculo de engaños: Excavación del cromlech de A Mourela (As Pontes de García Rodríguez, A Coruña): 163-175. Santiago de Compostela, Andavira.

Martín-Seijo, M.; Antolín, F.; Alonso, N.; Fábregas, R. y Bonilla, A. (2010): "Prácticas agrícolas y gestión del combustible en el monte gallego entre los siglos VII y XVII. El caso de A Mourela (As Pontes, A Coruña)", en A. M. S. Bettencourt, M. I.C. Alves y S. Monteiro-Rodrigues (eds.), Variações paleoambientais e evolução antrópica no Quaternário do Ocidente peninsular: 159-170. Braga, APEQ \& CITCEM.

Martín-Seijo, M.; Sartal Lorenzo, M.; Kaal, J. y TeiraBrión, A. (2018): “A Multi-Disciplinary Study of Woodcrafts and Plant Remains that Reveals the History of Pontevedra's Harbour (Northwest Iberia) Between the 13th and 19th Centuries AD". Environmental Archaeology doi: <https://doi.org/10.1 080/14614103.2018.1522782>.

Martínez-Cortizas, A.; Fábregas Valcarce, R. y Franco Maside, S. (2000): "Evolución del paisaje y actividad humana en el área de Monte Penide (Redondela, Pontevedra): Una aproximación metodológica". Trabajos de Prehistoria 57 (1): 173-184.

Martínez-Cortizas, A.; Mighall, T.; Pontevedra Pombal, X.; Novoa Muñoz, J.C.; Peiteado Varela, E. y Piñeiro, R. (2005): "Linking changes in atmospheric dust deposition, vegetation change and human activities in northwest Spain during the last 5300 years". The Holocene 15 (5): 698-706. doi: $<$ https://doi.org/10.1191/0959683605hl834rp>.

Martínez-Cortizas, A.; Pontevedra-Pombal, X.; García-Rodeja, E.; Novoa-Múñoz, J.C. y Shotyk, W. (1999): "Mercury in a Spanish peat bog: archive of climate change and atmospheric metal deposition". Science 284: 939-942. doi: <https://doi. org/10.1126/science.284.5416.939>.

McParland, L.C.; Collinson, M.E.; Scott, A.C.; Campbell, G. y Veal, R. (2010): "Is vitrification in charcoal a result of high temperature burning of wood?". Journal of Archaeological Science 37: 2679-2687. $<$ https://doi.org/10.1016/j.jas.2010.06.006>. 
Mira, J. A.; Carrión, Y. C.; Morales-Pérez, J. V. y Ortega, J. R. (2017): "Nuevas aportaciones sobre el aprovechamiento faunístico y vegetal en el Castell de Castalla (Alicante, España) durante los siglos XI-XV". Arqueología y Territorio Medieval 24: 291-330.

Moskal-del Hoyo, M.; Wachowiak, M. y Blanchette, R.A. (2010): "Preservation of fungi in archaeological charcoal". Journal of Archaeological Science 37 (9): 2106-2116. doi: <https://doi.org/10.1016/j. jas.2010.02.007>.

Ntinou, M.; Badal, E.; Carrión, Y.; Fueyo, J. L.M.; Carrión, R. F. y Mira, J. P. (2013): "Wood use in a medieval village: the contribution of wood charcoal analysis to the history of land use during the 13th and 14th centuries a. d. at Pobla d'Ifach, Calp, Alicante, Spain". Vegetation History and Archaeobotany 22 (2): 115-128. doi: <https://doi.org/10.1007/ s00334-012-0349-Z>.

Oliva, M.; Ruiz-Fernández, J.; Barriendos, M.; Benito, G.; Cuadrat, J. M.; Domínguez-Castro, F.; GarcíaRuiz, J.M.; Giralt, S.; Gómez-Ortiz, A.; Hernández, A.; López-Costas, O.; López-Moreno, J.I.; LópezSáez, J.A.; Martínez-Cortizas, A.; Moreno, A.; Prohom, M.; Saz, M.A.; Serrano, E.; Tejedor, E.; Trigo, R.; Valero-Garcés, B. y Vicente-Serrano, S.M. (2018): "The Little Ice Age in Iberian mountains". Earth-Science Reviews 177: 175-208. doi: <https:// doi.org/10.1016/j.earscirev.2017.11.010>.

Pardo de Guevara y Valdés, E.; Otero Piñeyro Maseda, P. S. y García González-Ledo, X. A. (2012): “La huella de los primeros Ulloa en las torres y fortalezas de la "terra" de Ulloa". Castillos de España 164-165-166: 59-67.

Pastoreau, M. (2006): Una historia simbólica de la Edad Media occidental. Buenos Aires, Ed. Katz.

Peña-Chocarro, L.; Pérez-Jordà, G.; Alonso, N.; Antolín, F.; Teira-Brión, A.; Tereso, J. P.; Montes Moya, E.; Reyes, D. L. (2019): "Roman and medieval crops in the Iberian Peninsula: a first overview of seeds and fruits from archaeological sites". Quaternary International 499-A: 49-66. doi: <https://doi. org/10.1016/j.quaint.2017.09.037>.

Py, V. y Durand, A. (2010): "Évolution des écosystèmes et des pratiques agrosylvopastorales et minière pour la production de bois de feu dans le haut Champsaur et la haute Durance (France) de l'âge du Bronze ancien au XVIe siècle", en Archéologie de l'espace montagnard: confrontation d'expériences européennes, Archéologie de la montagne européenne, Actes de la table ronde internationale de
Gap: 283-292. Gap (2008), Paris, Ed. Errance, col. BiAMA 4

Ríos Rodríguez, M. L. (1997): “Transformación agraria. Los terrenos de monte y la economía campesina (s. XII-XIV)", en M.P. de Torres Luna, R.C. Lois González y P. Saavedra (coord.), Semata: Ciencias sociais e humanidades. Espacios Rurais e Sociedades Campesiñas 9: 145-172.

Rodríguez-Ariza, M.O. (1993): “Los procesos de formación y transformación del registro arqueológico en los estudios antracológicos", en F. Burillo (ed.), Procesos postdeposicionales. Arqueología Espacial 16-17: 371-390.

Rouco, J. (2017): "Las funciones de una fortaleza bajomedieval: el caso de Pambre". Cuadernos de Estudios Gallegos 64 (130): 167-197.

Ruiz-Alonso, M.; Azkarate, A.; Solaun, J. L. y Zapata, L. (2012): "Exploitation of fuelwood in Gasteiz (Basque country, northern Iberia) during the Middle Ages (7001200 AD). Wood and charcoal evidence for human and natural history". Saguntum Extra 13: 227-236.

Santos, L.; Vidal, J.R. y Jalut, G. (2000): "History of vegetation during the Holocene in the Courel and Queixa Sierras, Galicia, northwest Iberian peninsula". Journal of Quaternary Science 15 (6): 621-632. doi: <https:// onlinelibrary.wiley.com/doi/abs/10.1002/1099$1417 \% 28200009 \% 2915 \% 3 \mathrm{~A} 6 \% 3 \mathrm{C} 621 \% 3 \mathrm{~A} \% 3 \mathrm{~A}$ AID-JQS524\%3E3.0.CO\%3B2-L>.

Schweingruber, F.H. (1990): Anatomy of European Woods. An atlas for the identification of European trees, shrubs and dwarf shrubs. Stuttgart, Paul Haupt.

Schweingruber, F.H. (2007): Wood Structure and Environment. Berlín-Heidelberg, Springer Verlag.

Schweingruber, F.H. y Börner A. (2018): The Plant Stem: A Microscopic Aspect. Berlín-Heidelberg, Springer Verlag.

Schmidt, O. (2006): Wood and tree fungi. Berlín-Heidelberg, Springer Verlag.

Scipioni S. (2002): "Dati antracologici da due pratiche attuali: la produzione del carbone vegetale nelle alte valli Scrivia e Trebbia (Appennino Ligure)". Archeologia Postmedievale 6: 49-65.

Shigo, A.L. (1979): Tree decay an expanded concept. Agriculture Information Bulletin 419: 1-72.

Shigo, A.L. y Marx, H.G. (1977): Compartmentalization of decay in trees. Agriculture Information Bulletin 405: 1-73.

Silva-Sánchez, N.; Martínez Cortizas, A. y López-Merino, L. (2014): "Linking forest cover, soil erosion and mire hydrology to late-Holocene human 
activity and climate in NW Spain". The Holocene 24 (6): 714-725.

Teira, A. (2013): “Dentro y fuera del bosque. La gestión del Prunus avium/cerasus en época romana y medieval en el NW ibérico". ArkeoGazte 3: 99-115.

Teira, A. (2015): "Cultivos e froiteiras na Idade Media en Galicia. O conxunto carpolóxico da escavación do Banco de España (Santiago de Compostela)". Gallaecia: Revista de Arqueoloxía e Antigüidade 34: 209-226.

Teira, A.M.; Martín-Seijo, M.; De Lombera-Hermida, A.; Fábregas, R. y Rodríguez-Álvarez, X.P. (2011): "Archaeobotanical analysis in sedimentation deposits of Roman and Medieval pits in caves of NW Iberia. Cova do Xato and Cova Eirós (Lugo, Galicia, Spain).”. Saguntum Extra 11: 163-164.

Théry-Parisot, I. (2001): Économie des combustibles au Paléolithique: Expérimentation, taphonomie, anthracologie. París, CNRS.

Théry-Parisot, I. y Henry, A. (2012): "Seasoned or green? Radial cracks analysis as a method for identifying the use of green wood as fuel in archaeological charcoal". Journal of Archaeological Science 39: 381-388. doi: <https://doi.org/10.1016/j. jas.2011.09.024>.

Tolksdorf, J. F.; Elburg, R.; Schröder, F.; Knapp, H.; Herbig, C.; Westphal, T.; Schneider, B.; Fülling, A. y Hemker, C. (2015): "Forest exploitation for charcoal production and timber since the
12 th century in an intact medieval mining site in the Niederpöbel Valley (Erzgebirge, Eastern Germany)". Journal of Archaeological Science: Reports 4: 487-500. doi: <https://doi.org/10.1016/j. jasrep.2015.10.018>.

Toriti, M. (2018): Les bois ouvragés durant l'Antiquité et le Moyen Âge en Gaule: approches interdisciplinaires depuis la coupe du bois jusqu'à la conservation du patrimoine ligneux. Tesis doctoral, Université du Maine. Inédita.

Traoré, M. (2018): Potential biomarkers of provenance of the wood from iberian typology shipwrecks (15th to 17th centuries). Tesis doctoral, Universidade de Santiago de Compostela. Inédita.

Traoré, M.; Kaal, J. y Cortizas, A. M. (2017): “Potential of pyrolysis-GC-MS molecular fingerprint as a proxy of Modern Age Iberian shipwreck wood preservation". Journal of Analytical and Applied Pyrolysis 126: 1-13. doi: <https://doi. org/10.1016/j.jaap.2017.07.003>.

Warren, G.; McDermott, C.; O’Donnell, L. y Sands, R. (2012): "Recent excavations of charcoal production platforms in the Glendalough valley, Co. Wicklow". Journal of Irish Archaeology 21: 85-112.

Zutter, C. (2000): "Wood and plant-use in 17th-19th century Iceland: archaeobotanical analysis of Reykholt, Western Iceland". Environmental Archaeology 5 (1): 73-82. doi: <https://doi.org/10.1179/ env.2000.5.1.73>. 
